chapters has a different author or authors, and there appears to have been little overall editorial control, so the contributions vary considerably in quality, purpose and organization. Few of them convey anything of the excitement, novelty or potential of research in this area.

The particular advantages of $C$. elegans are its neuroanatomy, development and genetics. The review of genetic analysis is excellent, and there arc also adequate chapters on developmental genetics, biochemical genetics and the molecular genetics of muscle. The section dealing with cell lineages and development is long and thorough, but needlessly turgid. Neuroanatomy is scarcely described at all (apart from a good general chapter on nematode sense organs), although the total anatomy and connectivity of most parts of the nervous system have been known for some time. This omission is especially unfortunate in view of the chapter on behaviour, and the admirable summary of work on Ascaris neurobiology. Thus there is still no opportunity of comparing the two species without going back to the original papers.

The second volume contains contributions on ageing and longevity, nutrition, respiration, osmoregulation, energy metabolism, and the structure of nematode cuticle and sense organs. Some of these chapters are accounts of nematological research, rather than the use of nematodes to investigate fundamental biological problems, and therefore seem inappropriate to these volumes. Nevertheless, there is a great deal of interesting material in the two books; they are up-todate and Academic Press has done a good job on the reproduction of photographs. For the time being, these volumes provide a useful introduction to contemporary research using nematodes.

J. Hodgkin is on the staff of the MRC Laboratory of Molecular Biology, Cambridge.

\title{
Genetics and plant populations
}

\author{
Donald A. Levin
}

Demography and Evolution in Plant Populations. Edited by Otto T. Solbrig. Pp.222. (Blackwell Scientific/University of California Press: 1980.) $£ 15, \$ 29.50$.

During the past decade, the discipline of plant demography crystallized from many disparate elements of empirical and theoretical ecology, and reproductive biology scattered in the basic and agricultural sciences. This discipline focuses principally on the quantitative aspects of germination, growth, reproduction, dispersal and death in a population, and the implications of these processes on the dynamics of population numbers and on life-history strategies. Although it is apparent that many characteristics of individuals related to the demography of populations are under genetic control, and that the demographic properties of populations may manifestly influence the genetic structure and amount and mobilization of genetic variation, the genetic perspective remains somewhat alien to scientists interested in plant demography. Demography and Evolution in Plant Populations is a step toward a unification of the ecological and evolutionary genetic components of demography.

The primary objective of the editor, Otto Solbrig, in assembling this collection of eight papers was to achieve a greater understanding of the dynamics of populations in relation to the action of natural selection. Papers by Abrahamson, Cook, Sarukhan and White deal almost exclusively with the dynamics of numbers of growth. One by Solbrig and another by
Lloyd are principally genetic in content. Only the paper by Snaydon broadly integrates genetic and numerical aspects of demography. The result is a volume about demography and about evolution, rather than about the reciprocal influences of numbers and genotypes in populations.

Although the volume does not provide a broad synthesis, it does contain informative treatments of some major topics in plant demography. The high points of the volume are the treatments of numbers and size in plants by White, and the genetic variable in demography by Snaydon. Both papers bring to light much agricultural information which ecologists and evolutionists are unlikely to encounter elsewhere. Sarukhan's contribution on demography in the tropics is also packed with new information and insight. In the other chapters, the biology of seeds in the soil is imaginatively reviewed by Cook; vegetative reproduction and the genetramet dichotomy are treated by Abrahamson; Lloyd discusses conditions favourable to self-fertilization in predominantly outbreeding species; and Solbrig reviews the genetic structure of populations, and highlights approaches which integrate demography and genetics.

Demography and Evolution in Plant Populations will be a useful and authoritative source of data and ideas about plant demography. Scientists interested in plant population dynamics, reproduction and growth will find the volume to be of considerable interest and value.

Donald A. Levin is Professor of Botany at the University of Texas, Austin, and specializes in plant evolution and ecological genetics.
Tanford number two

\section{B.A. Pethica}

The Hydrophobic Effect: Formation of Micelles and Biological Membranes. 2nd Edn. By Charles Tanford. Pp.233. (Wiley: 1980.) £10.50, \$24.65.

THIS second edition of Dr Tanford's wellknown book has been extensively rewritten to include recent advances in micelle studies, and contains a number of new chapters, most notably on serum lipoproteins and membrane proteins. It is not a review of recent results, but rather a synthesis or overview written in a lucid and occasionally magisterial style by a master of the subject.

The emphasis is on the phenomena of the hydrophobic effect, using thermodynamic arguments in a clear fashion which avoids pointless rigour. The accounts of light scattering, spectroscopic and similar methods are firm and uncluttered, and the recent rush of mathematical models receives sober treatment. The theme of the book reaches from simple solutions of amphipathic molecules to the structural basics of biomembranes, and in so slim a volume it would be difficult to please all readers with the choice of topics and facts. The omissions are almost as interesting as the inclusions. For example, bile salts are given short shrift, and the relation of dilute micellar solutions to the full phase diagrams is only touched upon. Wilkins et al. are quoted in support of the lipid bilayer model for erythrocytes, but Blaurock's reconsideration is not. Given that biomembranes are the sites of extensive chemical activity, the lack of reference to the now substantial body of work on micellar catalysis is a pity.

In the account of biomembranes it is good to see Robertson getting proper credit for his contribution to the bilayer membrane hypothesis. The now conventional view that biomembranes are fluid mosaics based on lipid bilayers is the one adopted by Dr Tanford, and to me the important last chapter (on membrane proteins) consequently has the air of skilful fitting of the hydrophobic proteins to a Procrustean bed. Perhaps when physicochemical data on the proteins are as extensive as for the lipids, the remarks of J.B.S. Haldane (quoted at the front of the book), which identify the membrane polymers as the key components, will be seen as truly prescient and the role of the lipids will be put into better perspective. $\mathrm{Be}$ that as it may, the hydrophobic effect will remain of major importance in the study of lipids and proteins alike, and this book will beneficially influence future research on membranes and other structures. We should hope for a third edition for the pleasure of reading Dr Tanford again.

B.A. Pethica is at Clarkson College, Potsdam, New York. 